\title{
Die Infrastruktur als Instrument traditioneller regionalpolitischer Strategien
}

\section{Ausgangslage}

Nach mehr als 10jähriger regionalpolitischer Tätigkeit wird zur Zeit vielerorts Bilanz gezogen (Anm. 1). Die Resultate erster Erfolgskontrollen zeigen, daß die regionalen Disparitäten zwischen prosperierenden Ballungsräumen und peripheren Entwicklungsregionen nicht kleiner, sondern eher noch größer geworden sind (STOEHR/TOEDTLING 1978, S. 90). Im vorliegenden Beitrag sollen die Unzulänglichkeiten traditioneller Förderstrategien aufgezeigt und mögliche Ansatzpunkte für eine alternative Regionalpolitik abgeleitet werden. Im Zentrum stehen dabei die Ausführungen über die Wirksamkeit der Infrastrukturpolitik im Rahmen mobilitätsorientierter Strategien. Einige generelle Bemerkungen zur grundsätzlichen Bedeutung des Infrastrukturbereichs für die regionalwirtschaftliche Entwicklung sollen für diese Diskussion die Basis bilden.

\section{Infrastruktur: Definition und wichtigste Eigenschaften}

In einer funktionalen Definition (Anm. 2) versteht JOCHIMSEN (1966, S. 145) unter Infrastruktur «die Gesamtheit aller materiellen, institutionalen und personalen Anlagen, Einrichtungen und Gegebenheiten, die den Wirtschaftseinheiten im Rahmen einer arbeitsteiligen Wirtschaft zur Verfügung stehen und die mit dazu beitragen,

(1) die Entgelte für gleiche Leistungen der Produktivkräfte auszugleichen und

(2) zugleich die größte Zuwachsrate der Gesamtwirtschaft herbeizuführen».

Diese weite Fassung des Infrastrukturbegriffs hat den Vorteil, den komplementären Charakter der verschiedenen Infrastrukturbereiche zu verdeutlichen. Ohne institutionale Infrastruktur ist die Wirkung der materiellen Infrastruktur weitgehend eingeschränkt. Die rechtliche und soziale Ordnung, Rechtsnormen sowie Traditionen - alles Elemente der institutionalen Infrastruktur - sind als Rahmenbedingungen für die regionalwirtschaftliche Entwicklung sehr wichtig. Ähnlich verhält es sich mit der personalen Infrastruktur, dem sogenannten «human capital», worunter die geistigen, unterneh- merischen und technischen Fähigkeiten der Wohnbevölkerung zu verstehen sind. Dieses Fähigkeitskapital ist eine entscheidende Voraussetzung für das Bestehen bzw. die Entwicklung hochspezialisierter, arbeitsteiliger Wirtschaftsformen. Wegen den vielfältigen, kurzfristigen Einsatzmöglichkeiten ist jedoch die materielle Infrastruktur für die regionale Entwicklungspolitik am bedeutendsten. Nach HIRSCHMAN (1967, S. 78) läßt sich der Bereich der materiellen Infrastruktur anhand folgender Merkmale kennzeichnen:

(1) Die Infrastruktur liefert die Grundlagen für die Produktion der anderen wirtschaftlichen Aktivitäten und für den Konsum. Sie ist damit notwendige Voraussetzung für räumliche und sektorale Arbeitsteilung und Spezialisierung.

(2) Die Infrastruktur wird in der Regel von der öffentlichen Hand erstellt oder zumindest von ihr kontrolliert.

(3) Infrastrukturelle Leistungen zeichnen sich durch einen hohen Grad an Standortgebundenheit aus. Es bestehen nur geringe Importmöglichkeiten.

(4) Infrastrukturinvestitionen sind durch technische Unteilbarkeiten und einen hohen Kapitalkoeffizienten gekennzeichnet.

Die Standortgunst einer Region wird in erheblichem Maße durch die Quantität und Qualität des zur Verfügung stehenden Angebots an materieller Infrastruktur geprägt. Durch Bereitstellung unternehmensorientierter Infrastrukturleistungen lassen sich wesentliche Quellen externer Effekte erschließen und damit die Produktionsbedingungen nachhaltig verbessern. Für die regionalwirtschaftliche Entwicklung sind jedoch nicht nur die in den Produktionsprozeß der Unternehmen eingehenden externen Vorteile der Infrastruktur von Bedeutung. Die Infrastruktur ist gleichzeitig eine entscheidende Determinante für die Wohnattraktivität und den Freizeitwert einer Region. Ein umfassender Ausbau der haushaltorientierten Infrastruktur ist in hochentwickelten Industriestaaten schon beinahe zur Selbstverständlichkeit geworden. Damit zeigt sich, $\mathrm{da} ß$ die strategische Bedeutung der Infrastruktur aus

Christian Hanser, Geographisches Institut Universität Zürich, 8033 Zürich. 
Abbildung 1: Wirkungen von Infrastrukturinvestitionen auf die Entwicklung einer Region.

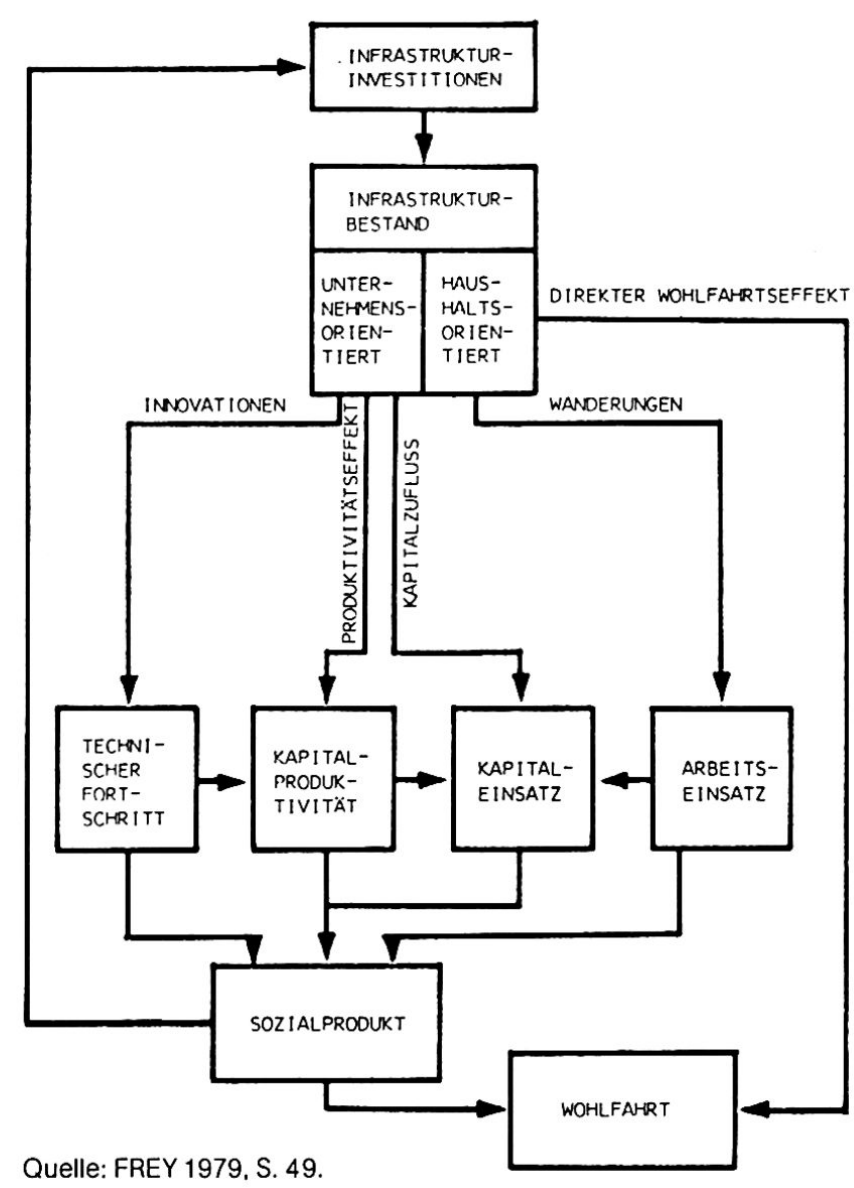

ihrer doppelten Wirkungsrichtung resultiert. Eine ausreichende Infrastruktur ist nicht nur erforderlich, um eine dynamische Entfaltung der regionalen Wirtschaft zu gewährleisten. Sie ist auch unabdingbare Voraussetzung für eine befriedigende demographische Entwicklung. Dabei entstehen Interdependenzen in der Art, daß eine Erhöhung des Wohnwertes gleichzeitig die Standortqualität und damit die Voraussetzungen für eine weitere industrielle Entwicklung verbessert, die dann wiederum auf die Einkommenssituation der Wohnbevölkerung rückwirkt (vgl. z. B. TOEPFER 1974). Diese generellen Zusammenhänge zwischen Infrastrukturausbau und wirtschaftlicher Entwicklung einer Region werden in Abbildung 1 zusammenfassend dargestellt.

\section{Die Stellung der Infrastrukturpolitik in mobilitätsorientierten regionalpolitischen Strategien}

Die Regionalpolitik westlicher Industrieländer ist im wesentlichen auf einen Ausgleich der Wohlfahrtsdisparitäten zwischen entwickelten (zentralen) und weniger entwickelten (peripheren) Regionen ausgerichtet. Arbeitswillige Staatsbürger sollen in der Region, in der sie ansässig sein wollen, eine ihrer Ausbildung entsprechende Beschäftigung finden und ein als ausreichend anzusehendes Einkommen erzielen können. Angesichts der erklärten Festlegung auf eine aktive Sanierung ist man sich weitgehend einig, daß der Weg zu einer langfristig sich selbst tragenden Entwicklung über die Schaffung gewerblicher und industrieller Arbeitsplätze führen muß. Damit wird einer Industrialisierung ländlich-peripherer Gebiete entscheidende Bedeutung beigemessen.

Den wichtigsten Engpaßfaktor für die wirtschaftliche Entwicklung peripherer Regionen in diese Richtung sieht man in der mangelnden Verfügbarkeit arbeitsplatzschaffender Unternehmen. Die bisherigen Formen der regionalen Wirtschaftspolitik beruhen deshalb im wesentlichen auf Kapitalhilfen (GANSER 1980, S. 7). Gemeinsames Kennzeichen ist die staatliche Subventionierung von privaten Investitionen, die in den benachteiligten Gebieten gewerbliche oder industrielle Dauerarbeitsplätze schaffen soll. Daneben erhalten aber auch die Gemeinden vom Staat Finanzhilfen für den Ausbau der wirtschaftsnahen (und/oder haushaltorientierten) Infrastruktur, um die Standortbedingungen für Industrieansiedlungen und Gewerbeerweiterungen $\mathrm{zu}$ verbessern. Auf kommunaler oder regionaler Ebene treten ergänzend Beiträge für die Erschließung und preisgünstige Abgabe von Industrieland hinzu.

Diese Maßnahmen bezwecken eine Kompensation von Produktionskostennachteilen peripherer Standorte. Durch Investitionssubventionierung und den Ausbau der produktionsrelevanten Infrastruktur sollen räumliche Investitionsalternativen zu den Ballungsgebieten geschaffen werden (FLORE 1978, S. 503). Mit der Verlagerung von Investitionen aus den überlasteten Verdichtungsräumen hinein in die Entwicklungsgebiete wird eine eigentliche interregionale Umverteilung von industriellen Produktionspotentialen angestrebt. Verschiedene Autoren sprechen in diesem Zusammenhang von mobilitätsorientierter Regionalpolitik oder einer Kapitalmobilisierungsstrategie (vgl. z. B. TOEPFER 1974, FUERST/ KLEMMER/ZIMMERMANN 1976).

Solche mobilitätsorientierte regionale Entwicklungsstrategien sind stark vom ökonomischen Denken neoklassischer Tradition beeinflußt (BUTTLER/ GERLACH/LIEPMANN 1977, S. 61). Die erhöhte Faktormobilität führt nach diesen Modellvorstellungen zu einer produktiveren Anordnung der Produktionsfaktoren im Raum. Die Produktionsfaktoren Arbeit und Kapital gelangen bei gewinnmaximalem Verhalten dort zum Einsatz, wo die größten Faktorentgelte erzielt werden können. Arbeitskräfte wandern von peripheren Regionen in die Ballungsgebiete, weil dort die Löhne höher sind. Das Kapital fließt in entgegengesetzter Richtung in die peripheren Gebiete hoher Kapitalproduktivität. Weiter wird argumentiert, daß sich hieraus ein interregionaler 
Ausgleich der Faktor- und Güterpreise ergebe, was dann eine Angleichung der regionalen Pro-KopfEinkommen zur Folge hätte. Mit der Intensivierung der räumlichen Integration bei gleichzeitiger Förderung der interregionalen Arbeitsteilung lassen sich nach neoklassischer Theorie zudem komparative Kostenvorteile erzielen, die sich schließlich in einem beschleunigten Wachstum der Gesamtwirtschaft niederschlagen (STOEHR/TOEDTLING 1977, S. 36).

In der traditionellen Regionalpolitik ist die neoklassische Doktrin nicht zuletzt deshalb so beliebt, weil sie von der Grundhypothese ausgeht, daß der Marktmechanismus von sich aus zu einem Ausgleich der unerwünschten regionalen Wohlstandsdisparitäten tendiert. Die Aufgabe des Staates beschränkt sich nach dieser Auffassung im wesentlichen darauf, möglichst günstige Rahmenbedingungen für die freie Entfaltung privater Initiativen zu schaffen (HUEBLER u.a. 1980, S. 51).

Vor diesem Hintergrund wird deutlich, daß dem Ausbau der Infrastruktur (vgl. Definition von JOCHIMSEN) im Rahmen einer mobilitätsorientierten Regionalpolitik eine zentrale Bedeutung beizumessen ist. Zur Erhöhung der Kapitalmobilität können insbesondere Verkehrsinvestitionen beitragen. Weitreichende Entwicklungseffekte erhofft man sich vor allem von der Einbindung peripherer Regionen in die Verkehrs- und Kommunikationsnetze benachbarter Ballungsgebiete und nationaler Metropolen. Standortnachteile werden auf diese Weise dadurch beseitigt, daß mit verbesserter Akzessibilität gewisse Agglomerationsvorteile «importiert» werden können und in umgekehrter Richtung der $\mathrm{Zu}$ gang zu den Nachfragezentren erleichtert wird. Bei immer knapper werdenden Bodenreserven in den Zentren unterstellt man aber auch Maßnahmen zur Erschließung von Industriegelände und den dazu gehörenden Ver- und Entsorgungseinrichtungen eine recht große Lenkungseffizienz (vgl. z. B. HANSER/ BUEHLER/KELLER 1980).

In neueren Strategien wird zusätzlich der Ausbau der haushaltorientierten Infrastruktur an die Hand genommen (Bildungs- und Gesundheitswesen, Freizeitinfrastruktur). Damit wird neben dem Standortentscheid industrieller Unternehmen auch der Wohnortentscheid der Arbeitskräfte zu einem expliziten Problem der Regionalpolitik. Dieser Ansatz ist primär darauf ausgerichtet, den für periphere Regionen so nachteiligen selektiven Abwanderungsprozessen entgegenzuwirken. Darunter wird die Tatsache verstanden, daß es eher die jüngeren, für die Entwicklung einer Region geeigneten und benötigten Arbeitskräfte sind, welche aus den Problemregionen abwandern, während die wenig dynamischen Personen mit tiefem Bildungsstand zurückbleiben (GATZWEILER 1975). Mit dem Verbleiben dieser qualitativ wertvollen Arbeitsmarktsegmente sollen zusätzlich günstige Rückkoppelungen auf die industrielle
Standortgunst erwirkt werden. Diesem Effekt wird zum Teil große Bedeutung beigemessen, da der Standortfaktor "ausreichendes Angebot an qualifizierten Arbeitskräften» mit Ausnahme der Zweigbetriebe meistens sehr hoch bewertet wird (FISCHER 1973, S. 79f.).

\section{Die Wirksamkeit mobilitätsorientierter regionalpolitischer Strategien}

\section{Mangelnde interregionale Lenkungseffizienz}

Die Resultate empirischer Untersuchungen zeigen indessen, daß mit der Infrastrukturpolitik im Rahmen mobilitätsorientierter Strategien trotz grundsätzlich positiven Bewertungen bei Wohnort- und Standortentscheidungen keine allzu großen Erfolge erzielt werden können. Infrastrukturmaßnahmen üben nämlich kaum interregionale Anreizwirkungen auf ansiedlungswillige Unternehmen aus. Das Angebot an Infrastrukturleistungen gehört in hochentwickelten Volkswirtschaften nicht mehr zu den Hauptdeterminanten der Standortwahl von Industriebetrieben (Anm.3). Die verkehrsmäßige Erschließung sowie die öffentliche Ver- und Entsorgung zu angemessenen Preisen zählen in der Regel zur Mindestausstattung eines Standorts und haben zudem weitgehend ubiquitären Charakter (FREY 1979, S. 108). Da Infrastrukturfaktoren bei der Feinbestimmung des Standorts aber doch von groBer Bedeutung sind, kann umgekehrt kaum mit einer industriellen Ansiedlung gerechnet werden, wenn diese Basisleistungen fehlen. FREY (1972, S. 40) prägte in diesem Zusammenhang das oft zitierte Schlagwort: Die Infrastruktur ist eine notwendige, jedoch keine hinreichende Voraussetzung für die wirtschaftliche Entwicklung einer Region. Die standortbeeinflussende Wirkung der Infrastruktur bleibt somit auf die kommunale oder höchstens regionale Ebene beschränkt.

Keine wesentlich anderen Effekte sind vom Ausbau der haushaltorientierten Infrastruktur zu erwarten. Die regionalen Präferenzen der Arbeitnehmer werden erst dann relevant und durch entsprechende Infrastrukturinvestitionen beeinflußbar, wenn eine befriedigende Einkommens- und Arbeitsplatzsituation gesichert scheint. Gerade in dieser Beziehung bieten die Ballungsräume aber nach wie vor die besseren Chancen - vor allem, wenn man qualitative Dimensionen wie Arbeitsplatzvielfalt oder Aufstiegsmöglichkeiten mitberücksichtigt. Zudem hat auch das Argument der Bildung von Arbeitskräftereserven an Kraft verloren. Die Nachfrage nach Arbeitskräften ist nach dem Konjunktureinbruch Mitte der 70er Jahre in vielen Industrieländern zurückgegangen und wird sich infolge zunehmendem Konkurrenzdruck aus den Niedriglohnländern eher noch weiter zurückbilden. 
Ebenfalls nur geringe interregionale Lenkungseffizienz weisen die im Rahmen von mobilitätsorientierten regionalpolitischen Strategien häufig verwendeten finanziellen Maßnahmen wie Steuererleichterungen oder Investitionsbeihilfen auf. Ähnlich wie die infrastrukturelle Basiserschließung hat auch die finanzielle Förderung lediglich den Stellenwert von einem unter mehreren Standortfaktoren. Maßnahmen der finanziellen Förderung werden erst dann entscheidungswirksam, wenn die weitergehenden, betriebsnotwendigen Mindestvoraussetzungen (Absatz/Bezug, Arbeitskräfte) an alternativen Standorten gegeben sind (FLORE 1976, S. 779). Ähnlich lautet die Beurteilung von BREDE (1971, S.93): "Vergleichsweise einflußreich scheinen finanzielle Hilfen erst dann zu sein, wenn sie die Gunst eines Standortes erhöhen, der primär andere Anforderungen erfüllt. Das heißt, standen zwei oder mehrere Standorte zur Wahl, die den vorrangigen Standortanforderungen genügten, dann fällt die Entscheidung zugunsten desjenigen Standorts aus, an dem der Unternehmer in den Genuß von Steuervorteilen oder öffentlicher Vergünstigung kommt.» Diese Ausführungen zeigen deutlich, daß die Standortdefizite peripherer Regionen mit dem hier beschriebenen Instrumentarium kaum zu beheben sind. Ein Vergleich wird trotz kompensatorischer Maßnahmen der Regionalpolitik zugunsten der großen Agglomerationen ausfallen. Die Möglichkeiten interregionaler Umverteilungen von industriellen Produktionspotentialen sind aus dieser Sicht als eher ungünstig zu beurteilen.

\section{Selektive Wirkungen der Industrieansiedlungs- politik}

Einige Länder melden nun trotzdem quantitative Erfolge ihrer Regionalpolitik (STOEHR/TOEDTLING 1978, S. 90f). Diese Ansiedlungserfolge sind grundsätzlich sicher als positiv zu bewerten. Sie werden nun allerdings relativiert, wenn man die durch regionalpolitische Förderung geschaffenen Arbeitsplätze auf qualitative Gesichtspunkte hin analysiert.

Investitionsbeihilfen und in geringerem Umfang auch infrastrukturelle Maßnahmen lösen selektive Wirkungen aus. In erster Linie werden Großbetriebe begünstigt, weil diese über eine größere Informationsverarbeitungskapazität verfügen und entsprechend umfangreichere Standortabklärungen durchführen. Die selektive Wirkung besteht aber auch in einer Begünstigung von Unternehmen mit geringen Standortanforderungen: Je geringere Ansprüche an das Qualifikationsniveau der Arbeitskräfte gestellt werden, desto eher reichen diese Maßnahmen zur Qualifizierung eines Standortes aus. Dies hat zur Folge, daß vor allem $Z$ weigbetriebe von Großbetrieben auf die Förderanreize reagieren und in peripheren Regionen Standort beziehen.
Der Ansiedlungstyp der Zweigbetriebe ist somit für die traditionelle Regionalpolitik sehr wichtig und stellt in vielen Fällen die einzige Möglichkeit zur Industrialisierung peripherer Regionen dar (FUERST/ ZIMMERMANN 1973, S. 173, MEIER/ELSASSER 1978, S. 16). Zweigstellengründungen zeichnen sich im Gegensatz zu Betriebsverlagerungen durch große Verlagerungsdistanzen aus, wobei die Tendenz zur Betriebsniederlassung in peripheren Regionen überwiegt (Anm. 4). Neugründungen sind für die Regionalpolitik von geringerer Bedeutung, da Standortalternativen außerhalb der Region des Gründerwohnsitzes meistens nicht in Frage kommen.

Diese Zweigbetriebe sind für die Entwicklung peripherer Regionen allerdings nicht unproblematisch, da sie oft eine einseitige Arbeitsplatzstruktur aufweisen. Sie bieten im allgemeinen kaum Ausbildungsplätze für Lehrlinge an; sie haben einen nahezu unbedeutenden Bedarf an Qualifikationen über Volksschulniveau, einen geringen Bedarf an Angestellten und qualifizierten Fachkräften und entsprechend hohe Frauenbeschäftigungsquoten. Sie vermehren bestenfalls das quantitative Arbeitsplatzangebot, schaffen jedoch keine neuen qualifizierten Arbeitsplätze, was eine wichtige Bedingung zur Umkehr oder Verringerung des Strukturtrends der dauernden Abwanderung qualifizierter und jugendlicher Arbeitskräfte wäre.

In engem Zusammenhang mit der ungenügenden Qualifikationsstruktur von Zweigbetrieben sehen BUTTLER/GERLACH/LIEPMANN (1975, S. 86) eine starke Konjunkturreagibilität dieses Ansiedlungstyps. Es wird dabei oft unterstellt, daß Zweigbetrieben im zyklischen Verlauf unternehmerischer Expansionsprozesse die spezifische Aufgabe der Abdeckung von Nachfragespitzen zugewiesen werde. Weiter wird argumentiert, daß bei Nachfragerückgang Freisetzungseffekte zuerst bei Zweigbetrieben auftreten. Bei anziehender Nachfrage seien unqualifizierte Arbeitskräfte auf dem externen Arbeitsmarkt ländlicher Regionen leicht rekrutierbar und wieder in den Produktionsprozeß einzubauen.

Mit einseitigen Bezugs- und Absatzbeziehungen lösen Zweigbetriebe zudem keine nennenswerten Attraktionswirkungen auf evtl. Verbund- und Folgeindustrien aus. Der nationale oder gar internationale Firmenzusammenhang enthebt die Zweigbetriebe des regionalen Integrationsbedarfs. "Konkret bedeutet dies, daß Rohstoffe und Zwischenprodukte in der Regel nicht in der Region bezogen werden, selbst wenn dies möglich wäre, sondern da, wo die Beschaffungszentrale des Konzerns die günstigste Möglichkeit ausgemacht hat» (METTLER-MEIBOM 1980, S. 278). Die Außenabhängigkeit und Fremdbestimmung der Region wird durch eine derartige Politik noch gefördert (STOEHR/TOEDTLING 1978, S. 93/95).

Aus solchen selektiven Wirkungen mobilitätsorien- 
tierter Förderstrategien können sich unter Umständen für periphere Regionen sehr nachteilige Konsequenzen ergeben. So weist z. B. EVERS (1976, S. 816) darauf hin, daß die Schaffung neuer mit der gleichzeitigen Vernichtung bestehender Arbeitsplätze zusammenfallen kann. Dies trifft insbesondere dann $\mathrm{zu}$, wenn Großindustrien und konzernabhängige Zweigwerke durch eine Vormachtstellung im örtlichen Arbeitsmarktgefüge wettbewerbsschwache, eingesessene Betriebe bei der Rekrutierung und Haltung von Arbeitskräften benachteiligen.

Wenn durch solche Industrieansiedlungen autochthone Unternehmen mit meist vielfältigen $\mathrm{Be}$ triebsfunktionen aus dem regionalen Arbeitsmarkt verdrängt werden, kann gar von eigentlichen Entleerungs- oder back-wash-Effekten gesprochen werden. Der damit einhergehende Verlust an Unternehmens- und Innovationspotential wiegt um so schwerer, als heute in diesem Bereich die eigentlichen Entwicklungsengpässe peripherer Regionen zu sehen sind (EWERS/WETTMANN 1978, S. 470). Qualitative Entleerungseffekte sind aber auch dann zu beobachten, wenn einerseits die regionale Bildungsinfrastruktur ausgebaut wird und gleichzeitig nur unqualifizierte Arbeitsplätze geschaffen werden (Anm. 5). Die mit zunehmendem Ausbildungsstand räumlich mobiler gewordenen Arbeitskräfte werden unattraktive Arbeitsmöglichkeiten nicht hinnehmen und abwandern. Es besteht somit die Möglichkeit, daß eine mobilitätsorientierte Regionalpolitik die selektiven Abwanderungsprozesse nicht verhindert, sondern im Gegenteil gar stimuliert (FESTER 1976, S. 136).

Eine ähnliche Entleerungshypothese kann auch im Zusammenhang mit der Anbindung benachteiligter Entwicklungsregionen an die Verkehrsnetze der Ballungsräume formuliert werden (vgl. z. B. GANSER 1980, FREY 1979). Eine Verkürzung der Reisezeiten bedeutet nämlich, daß die Pendlereinzugsbereiche der großen Agglomerationen weiter ausgreifen und die ohnehin viel zu kleinen Arbeitsmärkte peripherer Regionen weiter anfressen und auf diese Weise entwerten. Es wird zudem vermehrt Kaufkraft in die Agglomerationen abfließen, die bislang die in den benachteiligten Gebieten ansässigen Handels- und Dienstleistungsbetriebe gestützt hat. Schrittmacher beim Kaufkraftentzug sind die Einkaufszentren, in denen größere Warenmengen pro Einkauf beschafft werden, wofür sich dann ein weiterer Weg lohnt.

Im Zusammenhang mit solchen Entleerungseffekten, die unter Umständen durch die mobilitätsorientierte Regionalpolitik gar noch verstärkt werden, sind die auch in jüngster Zeit und trotz Regionalpolitik eher noch größer werdenden regionalen Disparitäten zu sehen (STOEHR/TOEDTLING 1978, S. 90).
Veränderte gesamtwirtschaftliche Rahmenbedingungen

Die Grundlagen für die traditionelle mobilitätsorientierte Regionalpolitik wurden in den 50er und 60 er Jahren erarbeitet, einer Zeit also, die durch hohe gesamtwirtschaftliche und industrielle Wachstumsraten gekennzeichnet war. Aufgrund dieser Strategien soll ein Disparitätenabbau dadurch zustande kommen, daß die wachstumsbegleitenden und -induzierenden industriellen Investitionen durch geeignete regionalpolitische Maßnahmen in die Problemgebiete umgelenkt werden. Die bisherige Regionalpolitik war somit auf eine Umverteilung zukünftiger Mobilitätspotentiale ausgerichtet.

Seit Beginn der 70er Jahre steht nun allerdings der industrielle Sektor der meisten Industrieländer vor erheblichen Anpassungsproblemen. Die Verknappung und Verteuerung der Rohstoffe, die ständig veränderten Währungsrelationen und damit einhergehende Exporterschwernisse sowie die weltweite Verlagerung technisch ausgereifter, arbeitsintensiver Massenproduktion in Niedriglohnländer brachten das industrielle Wachstum beinahe zum Erliegen. Mit den auf diese Weise veränderten gesamtwirtschaftlichen Rahmenbedingungen ist die Manövriermasse für eine mobilitätsorientierte Regionalpolitik stark geschrumpft. Die Erfolgschancen einer auf Mobilitätspotentialen aufbauenden Regionalpolitik werden dadurch empfindlich eingeschränkt (FLORE 1978, S. 504; BRUGGER 1980, S. 4).

\section{Schlußfolgerungen}

Die Maßnahmen einer mobilitätsorientierten Regionalpolitik bezwecken im wesentlichen eine Kompensation von Produktionskostennachteilen peripherer Standorte. Dadurch soll eine interregionale Umverteilung industrieller Produktionspotentiale in die Wege geleitet und eine Angleichung regionaler ProKopf-Einkommen erreicht werden.

Ob dieses Ziel mit dieser stark auf neoklassischem Gedankengut basierenden und auf funktionale Integration ausgerichteten Strategie erreicht werden kann, muß allerdings stark bezweifelt werden. Die wichtigsten Kritikpunkte ergeben sich im Anschluß an die vorhergehende Wirkungsanalyse aus der mangelnden interregionalen Lenkungseffizienz des eingesetzten Instrumentariums und damit verbunden aus den selektiven Förderwirkungen mit einseitiger Bevorzugung von Zweigbetrieben mit standardisiertem Produktionsprogramm. Es besteht zudem eine nicht zu unterschätzende Gefahr, daß durch die praktizierte Ansiedlungs- und Infrastrukturpolitik kumulative Entleerungseffekte erzeugt oder verstärkt werden. Entscheidend geschmälert werden die Erfolgschancen einer auf Mobilitätspotentialen auf- 
bauenden Regionalpolitik aber auch durch die Veränderung der gesamtwirtschaftlichen Rahmenbedingungen. Regionalpolitische Manövriermassen bestehen infolge niedriger industrieller Wachstumsraten praktisch nicht mehr.

Aus der hier vorgebrachten Kritik kann mit aller Vorsicht gefolgert werden, daß eine alternative $R e$ gionalpolitik stärker auf die Erfordernisse einer "Entwicklung von innen» ausgerichtet werden müßte. Damit wäre insbesondere die bestmögliche Förderung des bestehenden, endogenen Potentials anzustreben. Innovationsorientierten Maßnahmen muß in diesem Zusammenhang eine hohe Priorität beigemessen werden. Ein besonderes Anliegen muß einer alternativen Regionalpolitik aber auch die Entwicklung und Fortbildung der einheimischen Wirtschaftseliten sein, um auf diesem Weg die Innovations- und Konkurrenzfähigkeit der Region zu stärken. Erforderlich wären ferner ein $A b b a u$ der gro $\beta$ räumigen Integration und die gleichzeitige Verbesserung der innerregionalen Erschließung sowie die Förderung der Arbeitsteilung zwischen peripheren Regionen. Infrastrukturelle Maßnahmen sind auch im Rahmen einer alternativen Regionalpolitik von großer Bedeutung, allerdings unter veränderten Prämissen des Mitteleinsatzes.

\section{Anmerkungen}

(1) Unter Regionalpolitik und synonym dazu regionaler Wirtschaftspolitik wird in Anlehnung an FUERST u.a. (1976, S. 91) die bewußte Gestaltung der räumlichen Struktur einer Volkswirtschaft verstanden.

(2) Wegen der Heterogenität des Infrastrukturbereichs behilft man sich anstelle einer funktionalen Definition oft mit einer kasuistischen Zuordnung eines Sachbereichs zur Infrastruktur. Vgl. z. B. den Aufsatz von CHR. BECKER in diesem Heft.

(3) Als Hauptdeterminanten der industriellen Standortwahl können die Faktoren Marktnähe und Wachstum des Marktes, Rohstoffbezug, der Boden, die Arbeitskräfte und die Verkehrsverhältnisse bezeichnet werden. Vgl. hierzu die zusammenfassende Darstellung von FREUND/ZABEL (1978, S. 126).

(4) Der Anlaß zu einer Betriebsverlagerung ergibt sich meist aus ungenügenden Grundstückverhältnissen. Da im Durchschnitt nicht mehr als 10 bis $20 \mathrm{~km}$ zurückgelegt werden, handelt es sich meist um Stadtrandwanderungen (BADE 1978, S. 23).

(5) Vgl. hierzu den Aufsatz von E. BUEHLER-CONRAD in diesem Heft.

\section{Literaturverzeichnis}

BADE, FRANZ-JOSEF: Sektorale und räumliche Aspekte der Mobilität industrieller Betriebe, IIMpaper Nr. 78-2, Berlin 1978.

BREDE, HELMUT: Bestimmungsfaktoren industrieller Standorte, Schriftenreihe des Ifo-Institutes für Wirtschaftsforschung München Bd. 75, Berlin 1971.

BRUGGER, ERNST A.: Regionale Anpassungsschwächen und innovationsorientierte Regionalpolitik, Manuskript, Bern 1980.

BUTTLER, FRIEDRICH, GERLACH, KNUT, LIEPMANN, PETER: Funktionsfähige regionale Arbeitsmärkte als Bestandteil ausgewogener Funktionsräume, in: Veröffentlichungen der Akademie für Raumforschung und Landesplanung, Forschungs- und Sitzungsberichte Bd. 94, Hannover 1975.

BUTTLER, FRIEDRICH, GERLACH, KNUT, LIEPMANN, PETER: Grundlagen der Regionalökonomie, Reinbeck bei Hamburg 1977.

EVERS, ADALBERT: Zur Wirksamkeit der regionalen Wirtschaftsförderung als Entwicklungs- und Notstandspolitik, in: Informationen zur Raumentwicklung, Heft 12, 1976.

EWERS, HANS-JURGEN, WETTMANN, REINHART: Innovationsorientierte Regionalpolitik - Überlegungen zu einem regionalstrukturellen Politik- und Forschungsprogramm, in: Informationen zur Raumentwicklung, Heft 7, 1978.

FESTER, FLORIAN: Entwicklungszentren - Urbanisierung peripherer Regionen statt Industrialisierung des ländlichen Raums, in: Informationen zur Raumentwicklung, Heft 2/3, 1976.

FISCHER, GEORGES: Praxisorientierte Theorie der Regionalforschung, Tübingen 1973.

FLORE, CARL: Regionale Wirtschaftspolitik unter veränderten Rahmenbedingungen, in: Informationen zur Raumentwicklung, Heft 12, 1976.

FLORE, CARL: Instrumente der Innovationsförderung im Rahmen der Raumordnungspolitik, in: Informationen zur Raumforschung, Heft 7, 1978.

FREUND, ULRICH, ZABEL, GERHARD: Regionale Wirkungen der Wirtschaftsstrukturförderung, Schriftenreihe "Raumordnung» des Bundesministers für Raumordnung, Bauwesen und Städtebau 06.023, Bonn 1978.

FREY, RENÉ L.: Infrastruktur - Grundlagen der Planung öffentlicher Investitionen, Tübingen und Zürich 1972, 2. Auflage.

FREY, RENÉ L.: Die Infrastrukturpolitik als Mittel der Regionalpolitik, Publikationen des Schweizerischen Nationalfonds aus den Nationalen Forschungsprogrammen Bd. 1, Bern und Stuttgart 1979. 
FUERST, DIETRICH, KLEMMER, PAUL, ZIMMERMANN, KLAUS: Regionale Wirtschaftspolitik, wisu-texte, Tübingen 1976.

FUERST, DIETRICH, ZIMMERMANN, KLAUS: Standortwahl industrieller Unternehmen, Schriftenreihe der Gesellschaft für regionale Strukturpolitik Bd.1, Bonn 1973.

GANSER, KARL: Die «Sonderaktion der österreichischen Bundesregierung zur Stärkung entwicklungsschwacher ländlicher Räume im Berggebiet» - Ein Kommentar, in: Informationen zur Raumentwicklung, Heft 1, 1980.

GATZWEILER, HANS-PETER: Zur Selektivität interregionaler Wanderungen, Forschungen zur Raumentwicklung Bd. 1, Bonn 1975.

HANSER, CHRISTIAN, BUEHLER, ELISABETH, KELLER, THEO: Methodische Ansätze zur Wirkungskontrolle der Infrastrukturpolitik, Arbeitsberichte des Nationalen Forschungsprogramms «Regionalprobleme in der Schweiz» Nr. 11, Bern 1980.

HIRSCHMAN, ALBERT O.: Die Strategie der wirtschaftlichen Entwicklung, Stuttgart 1967 (dt. Ausgabe).

HUEBLER, KARL-HERMANN u.a.: Zur Problematik der Herstellung gleichwertiger Lebensverhältnisse,
Veröffentlichungen der Akademie für Raumforschung und Landesplanung, Abhandlungen Bd. 80, Hannover 1980.

JOCHIMSEN, REIMUT: Theorie der Infrastruktur Grundlagen der marktwirtschaftlichen Entwicklung, Tübingen 1966.

MEIER, RUEDI, ELSASSER, HANS: Die Industriepolitik im Wallis - eine empirische Untersuchung zur Erfolgskontrolle, Manuskript, Zürich 1978.

METTLER-MEIBOM, BARBARA: Grundzüge einer regionalen Regionalpolitik, in: Informationen zur Raumentwicklung, Heft 5, 1980.

STOEHR, WALTER, TOEDTLING, FRANZ: Spatial Equity - some Anti-theses to Current Development Doctrine, in: Papers of the Regional Science Association, vol 38, 1977.

STOEHR, WALTER, TOEDTLING, FRANZ: An Evaluation of Regional Policies - Experiences in Marked and Mixed Economies, in: HANSEN NILES M. (ed.) Human Settlement Systems, International Perspectives on Structure, Change and Public Policy, Cambridge, Massachusetts 1978.

TOEPFER, KLAUS: Standortentscheidung und Wohnortwahl, Kleine Schriften der Gesellschaft für regionale Strukturentwicklung, Bonn 1974.

\section{Literaturbesprechung}

SENNHAUSER, Robert u.a.: Ortsplanung heute für morgen - Eine kleine Planungsfibel für Behördenmitglieder und Politiker. Eidgenössische Zukunft Heft 15. Verlag Paul Haupt Bern 1979. 112 Seiten, kartoniert, Fr. 19.--

Diese Planungsfibel will den verantwortlichen Behördemitgliedern und Politikern Denkanstösse - aber keine Rezepte - geben, wie sie die Aufgaben der Ortsplanung praktisch anpacken können. In der Gemeinde wird nicht nur die Raumplanung in die Realität ungesetzt; die Gemeindeebene ist zugleich die geeignetste Plattform für die Beteiligung der Bürger an der Planung. In diesem Sinne richtet sich die Planungsfibel auch an die interessierten Bürger.
Der Mange1 an Lehrbüchern über die schweizerische Raumplanung auf allen Stufen wird zu Recht immer wieder - vor allem von Seiten der Geographielehrer - beklagt. Mit der vorliegenden Planungsfibel ist es den Verfassern gelungen, diese Lücke teilweise zu schliessen. Es ist zu hoffen, dass 'Ortsplanung heute für morgen' in breitem Masse im Geographieunterricht Verwendung findet. Aber auch dem Geographiestudenten kann dieses Buch bestens empfohlen werden. Die Geographie darf sich ja nicht auf die Analyse unseres heutigen Lebensraumes beschränken, sondern sie muss sich auch mit dem Lebensraum künftiger Generationen und seiner Gestaltung auseinandersetzen.

GH $4 / 80$

Hans E1sasser, Zürich 